Proceedings of the 2nd International Congress APMAS2012, April 26-29, 2012, Antalya, Turkey

\title{
Multi-Electrode Tapered Waveguide Structure for Quantum-Dot Semiconductor Optical Amplifiers
}

\author{
K. ABedi* And E. MohadesRad \\ Department of Electrical Engineering, Faculty of Electrical and Computer Engineering \\ Shahid Beheshti University, G.C. 1983963113, Tehran, Iran
}

\begin{abstract}
In this paper, by employing two practical methods of multi-electrode design and tapered waveguide structure, compensation techniques to restore declining carrier density along the quantum dot semiconductor optical amplifier's waveguide are investigated. In the multi-electrode method, the effects of distributing current via electrodes on the gain and the cross-gain modulation for two, three and four-electrode structures are studied. These characteristics are also investigated for the quantum dot semiconductor optical amplifiers with a tapered waveguide structure for the first time, including waveguides with different profiles and width ratios. After due comparison between these two methods, optimum parameters of the two techniques will be used simultaneously along each other to form a combined method. Through these design developments, gain as the main feature of the quantum dot semiconductor optical amplifiers continuously increases, though cross-gain modulator results show that there is a trade-off between the modulation efficiency and improving structure capability of restoring carrier density.
\end{abstract}

DOI: $10.12693 /$ APhysPolA.123.411

PACS: 42.50.-p, 42.30.Lr, 42.79.-e

\section{Introduction}

Carrier density reduction along the waveguide, resulting from the stimulated emission caused by the propagating optical signal, imposes a locally varying and declining gain. In other words, the optical gain of the quantum-dot semiconductor optical amplifier (QD-SOA) along the active region is nonlinearly decreasing [1-5]. Theoretically in order to restore the decreased carrier density in the far side of the QD-SOA, a nonlinear profile of current injection can be applied, providing that the profile dictates a nonuniform increasing current [6]. Practically, the nonuniform current injection can be realized through multi-electrode QD-SOAs [7]. In such devices, different currents are conducted via two or more electrodes into the waveguide in a manner in which that the end of the device, where the carrier density is largely compromised, will receive more current. For avoiding complicated driving circuits, especially for more than two electrodes, and other difficulties which arise with implementing several electrodes, the tapered waveguide approach can be employed [8-10]. In this study, at first two methods of tapered waveguide and multi-electrode structure are investigated and compared to each other with regard to optical gain and cross-gain modulation (XGM). Next we will study using these two methods simultaneously together to see if the desired characteristics can be enhanced.

The exact QD-SOA that we have employed in our study as well as the rate equations which explain its behavior have been introduced in Ref. [6]. Summary of the main equations governing the rate of variation in the energy states' occupation probabilities is mentioned as follows:

*corresponding author; e-mail: $\mathrm{k}_{-}$abedi@sbu.ac.ir

$$
\begin{aligned}
\frac{\partial f_{i}^{n}}{\partial t} & =\left(R_{i+1, i}^{n c}-R_{i, i+1}^{n e}\right)-\left(R_{i, i-1}^{n c}-R_{i-1, i}^{n e}\right) \\
- & R_{i}^{s p}-R_{i}^{s t}, \\
\frac{\partial f_{k}^{p}}{\partial t} & =\left(R_{k+1, k}^{p c}-R_{k, k+1}^{p e}\right)-\left(R_{k, k-1}^{p c}-R_{k-1, k}^{p e}\right), \\
\frac{\partial w_{p}}{\partial t} & =\frac{I}{q V_{a} N_{\mathrm{WL}}}-\left(R_{w p, 7}^{n c}-R_{7, w p}^{n e}\right)-R_{w p}^{s p} \\
+ & D_{p} \frac{\partial^{2} w_{p}}{\partial z^{2}}, \\
\frac{\partial w_{n}}{\partial t} & =\frac{I}{q V_{a} N_{\mathrm{WL}}}-\left(R_{w_{n}, 2}^{n c}-R_{2, w_{n}}^{n e}\right)-R_{w_{n}}^{s p} \\
+D_{n} \frac{\partial^{2} w_{n}}{\partial z^{2}} & \\
R_{i}^{s p} & =\frac{f_{i}^{n} f_{i}^{p}}{\tau_{i R}}\left(\alpha_{i i}^{n}+c_{i i}^{p} f_{i}^{p}+c_{i i}^{n} f_{i}^{n}\right), \\
R_{i}^{s t} & =\frac{v_{g} g_{i}}{N_{\mathrm{Q}}}\left(f_{i}^{p}+f_{i}^{n}-1\right) S, \\
\frac{\partial S}{\partial z} & =g_{\mathrm{QD}} S-\alpha S, \\
g_{\mathrm{QD}} & =\sum_{j=0}^{H} g_{j}\left(f_{j}^{n}+f_{j}^{p}-1\right) .
\end{aligned}
$$

\section{Multi-electrode QD-SOA}

The basic concept of the multi-electrode method is illustrated in Fig. 1. In every QD-SOA with an injection current that increases monotonically along the waveguide, as long as optimizing the current's parameters is considered, reaching the optimum gain is possible [6]. So according to Ref. [6], we have chosen exponential function 


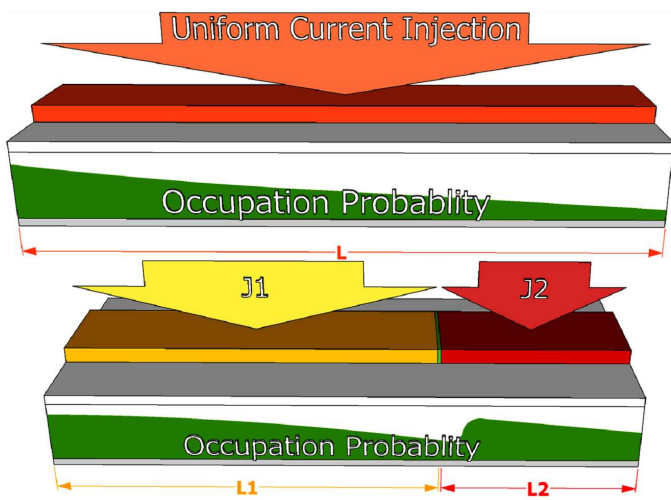

Fig. 1. Schematic of two-electrode structure.

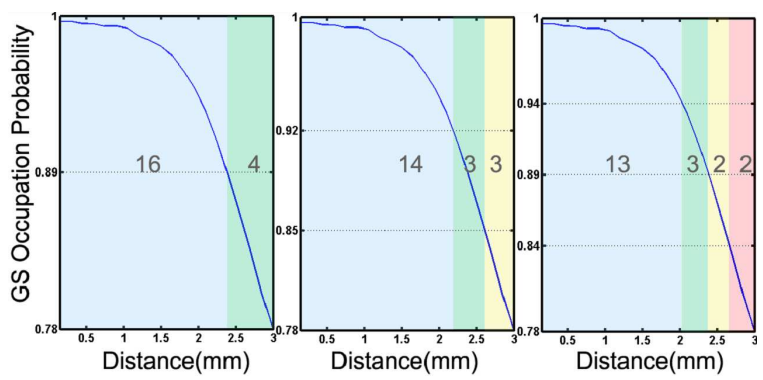

Fig. 2. The dashed line(s) represents the $50 \%, 33 \%$ and $25 \%$ drop along the active region in the occupation probability of the conduction bands (CB's) ground state.

in the form of $J(z)=b \exp (a z)$ for the current's profile. In that equation $b=0.8 J_{0}$ and parameter $a$ is calculated so that the number of injected carriers to the device will be equal to the number of injected carriers when the current's profile is uniform. The only issue that remains is choosing the lengths of the electrodes. Here, we should mention that the method, by which the lengths of the electrodes had been chosen by an earlier study on two-electrode structure [7], is unclear. In our study, taking into consideration variation of the ground-state

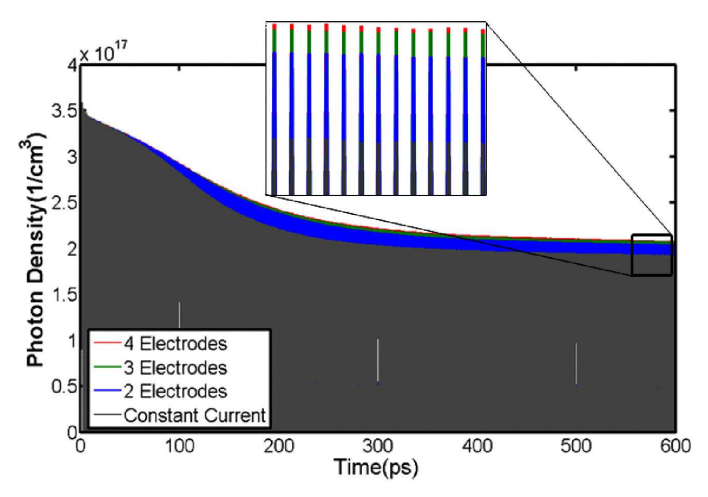

Fig. 3. The output photon density for generic QD-SOA and two, three and four-electrode structures.

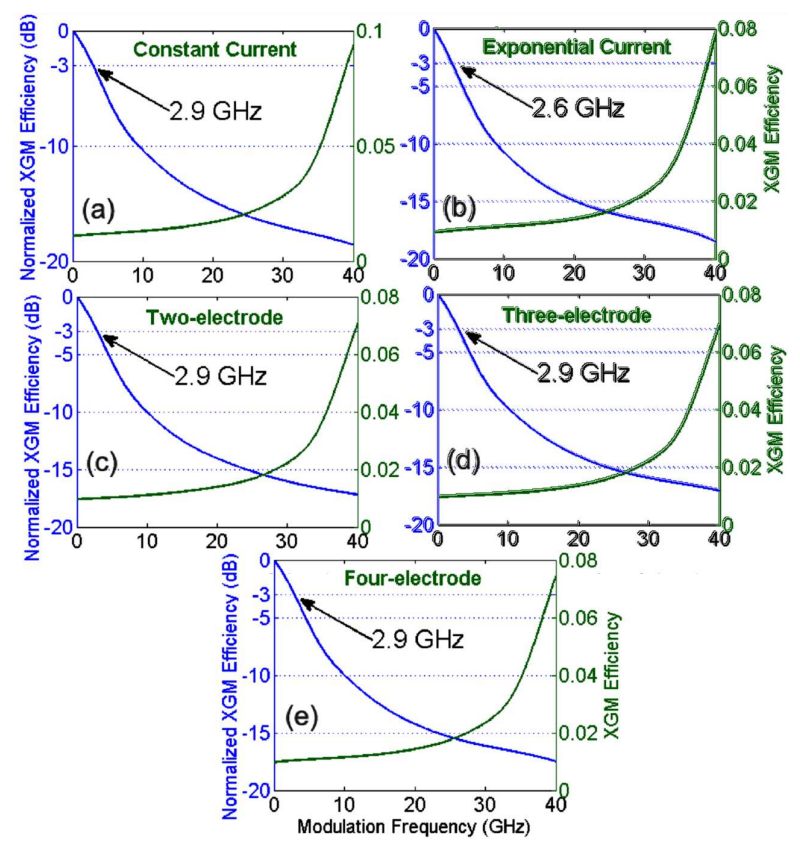

Fig. 4. Normalized XGM efficiency (blue) and XGM efficiency (green).

occupation probability along the active region while injecting constant current, the proportional lengths for two, three and four-electrode structures are chosen as $(16,4)$, $(14,3,3),(13,3,2,2)$, respectively (Fig. 2).

The output photon densities for multi-electrode structures and constant current structure are depicted in Fig. 3. By going through two, three and four-electrode methods the output photon density approaches the optimum photon density resulting from the non-uniform current, in a manner that ultimately the photon density of the four-electrode structure becomes indistinguishable from the exponential current's. XGM efficiencies and normalized XGM efficiencies for all the cases that have been discussed so far, which cover constant current, exponential current, two, three and four-electrode QD-SOAs, are depicted in Fig. 4.

\section{Tapered waveguide structure}

A basic structure for a tapered QD-SOA is illustrated in Fig. 5. Figure 6 shows the effect of increasing the output-to-input width ratio of an exponentially tapered waveguide on the modal gain. Results for different shapes of the tapered structure are demonstrated in Fig. 7, which shows that the gains of all different shapes have almost the same pattern, and as the output-to-input width ratio increases these patterns become more indistinguishable. It should be considered that all the waveguides reach the same width at the end. The XGM results for the tapered structure are depicted in Fig. 8. These results make evident that we may limit the waveguide's output-to-input width ratio to two so that the device will not 


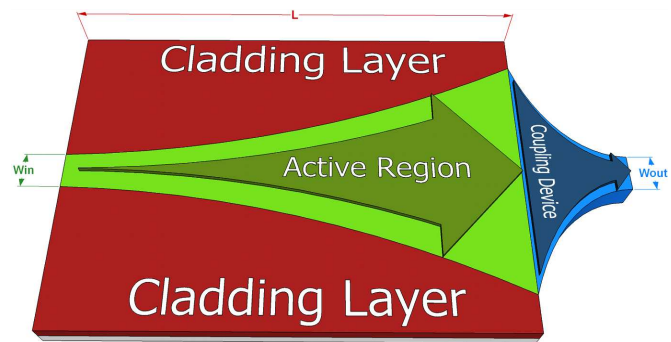

Fig. 5. Schematic diagram of a QD-SOA with a tapered waveguide structure attached to a coupling device in order to return the waveguide to its initial width.

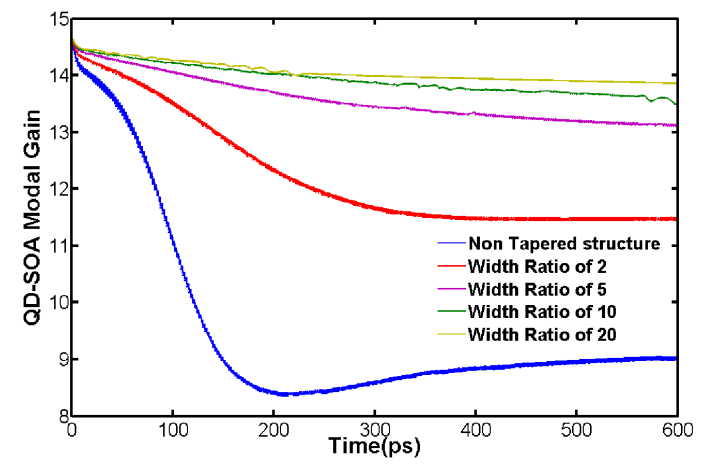

Fig. 6. Tapered QD-SOA modal gain for different width ratios obtained at the end of the device.

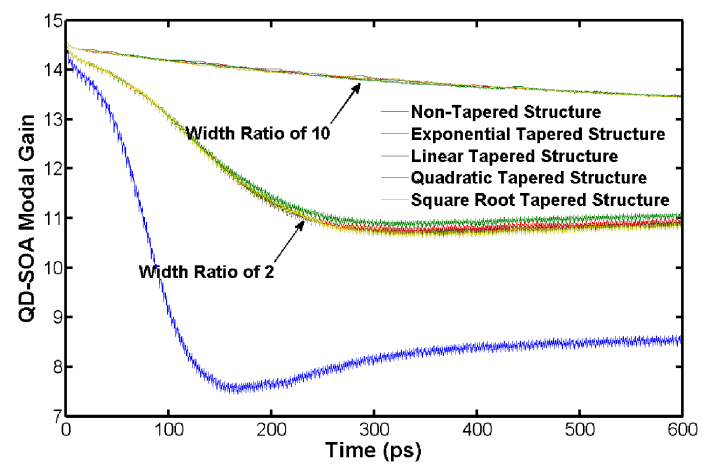

Fig. 7. The modal gain of the tapered QD-SOA for different shapes of the waveguide with width ratios of two and ten, obtained at the end of the device.
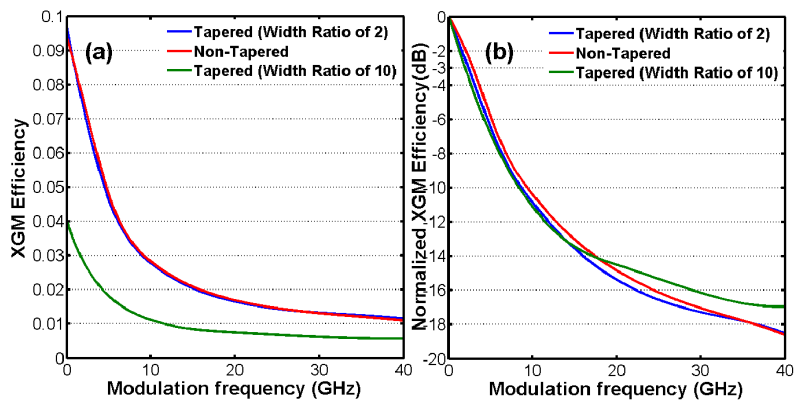

Fig. 8. XGM efficiency (a) and normalized XGM efficiency (b). lose its XGM efficiency substantially. Moreover, we will secure a decent gain for our tapered QD-SOA.

\section{Combined method}

Schematic diagram of the method formed by combining tapered waveguide and two-electrode structure is illustrated in Fig. 9. Since the major increase in gain occurs with two-electrode structure and also for maintaining the practicality of this method, we have limited the number of electrodes for this structure to two. So the combined

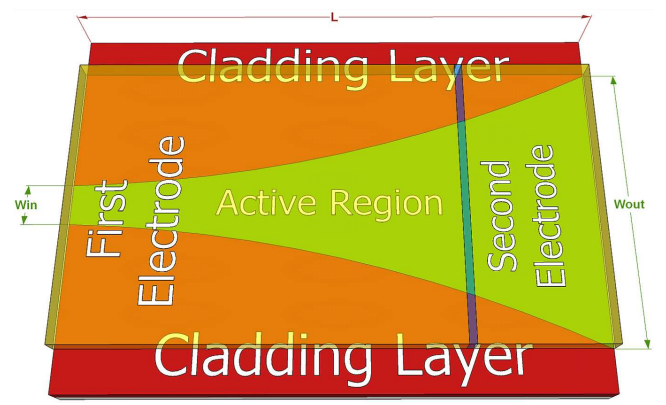

Fig. 9. Schematic diagram of a method formed from tapered waveguide and two-electrode QD-SOA.

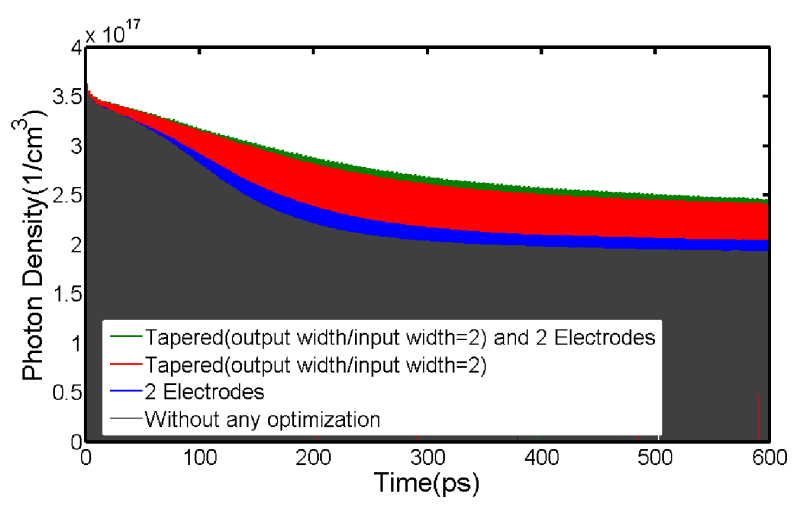

Fig. 10. The output photon density for the generic QD-SOA, two-electrode, tapered and the combined methods.
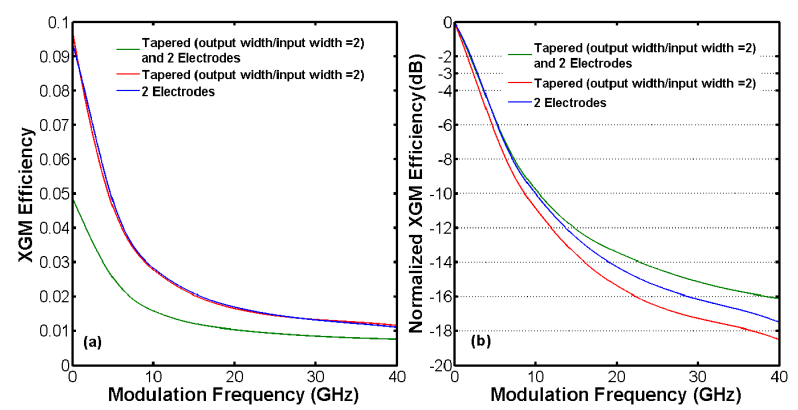

Fig. 11. XGM efficiency and normalized XGM efficiency results of the combined method. 
structure is shaped from the tapered waveguide structure with output-to-input width ratio of two and has a two-electrode metal contact. The results of the combined method are depicted in Figs. 10, 11.

\section{Conclusions}

In this paper, the gain and the XGM of QD-SOAs with multi-electrode and tapered waveguide structures were investigated. As we went through different methods, including uniform current injection, multi-electrode structures, tapered waveguide, and the combined method, the attainable gain steadily increased. In multi-electrode method the major gain improvement occurred by employing two-electrode structure, and subsequently increasing the number of electrodes to four resulted in approaching the optimum gain which has been reached by applying non-uniform current. The XGM however, had a completely different behavior so it did not change significantly by our structural developments or in the cases of extreme widening of the tapered waveguides or forming the combined method, even decreased. These drops show that there is a trade-off between the structure's ability to restore compromised carrier density and the modulation efficiency.

\section{References}

[1] H. Schmeckebier, C. Meuer, D. Bimberg, C. Schmidt-Langhorst, A. Galperin, C. Schubert, Semicond. Sci. Technol. 26, 014009 (2011).

[2] S. Lv, I. Montrosset, M. Gioannini, S. Song, J. Ma, Optoelectron. Lett. 7, 122 (2011)

[3] A. Rostami, H. Nejad, R.M. Qartavol, H.R. Saghai, IEEE J. Quant. Electron. 46, 354 (2010).

[4] G. Contestabile, A. Maruta, K. Kitayama, IEEE Photon. Technol. Lett. 22, 987 (2010).

[5] O. Qasaimeh, J. Lightwave Technol. 27, 1978 (2009)

[6] H. Taleb, K. Abedi, S. Golmohammadi, Appl. Opt. 50, 608 (2011)

[7] Y. Yi, H. Lirong, X. Meng, T. Peng, H. Dexiu, J. Opt. Soc. Am. B 27, 2211 (2010)

[8] X. Wang, G. Erbert, H. Wenzel, B. Eppich, P. Crump, A. Ginolas, J. Fricke, F. Bugge, M. Spreemann, G. Tränkle, Semicond. Sci. Technol. 27, 015010 (2012)

[9] D. Skoczowsky, A. Jechow, R. Menzel, K. Paschke, G. Erbert, Opt. Lett. 35, 232 (2010).

[10] W.M. Wong, H. Ghafouri-Shiraz, IEEE J. Sel. Top. Quant. Electron. 6, 585 (2000) 